\title{
Adropin and irisin: New biomarkers of cardiac status in patients with end-stage renal disease? A preliminary study
}

\author{
Małgorzata Kałużna ${ }^{1, A-D, F}$, Krzysztof Pawlaczyk ${ }^{2, A, C-F}$, Krzysztof Schwermer, ${ }^{2, B-D, F}$, Krzysztof Hoppe ${ }^{2, B, F}$, \\ Magdalena Człapka-Matyasikik, ${ }^{3, B-F}$, Aisha Yusuf Ibrahim ${ }^{2, D-F}$, Nadia Sawicka-Gutaj ${ }^{1, B, E, F}$,

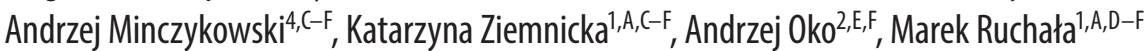 \\ ${ }^{1}$ Department of Endocrinology, Metabolism and Internal Diseases, Poznan University of Medical Sciences, Poland \\ 2 Department of Nephrology, Transplantology and Internal Diseases, Poznan University of Medical Sciences, Poland \\ ${ }^{3}$ Department of Human Nutrition and Hygiene, Poznan University of Life Sciences, Poland \\ ${ }^{4}$ Department of Cardiology - Intensive Therapy, Poznan University of Medical Sciences, Poland \\ A - research concept and design; B - collection and/or assembly of data; C - data analysis and interpretation; \\ $D$ - writing the article; $E$ - critical revision of the article; $F$ - final approval of the article
}

Address for correspondence

Krzysztof Pawlaczyk

E-mail:kpawlac@ump.edu.pl

Funding sources

None declared

Conflict of interest

None declared

Received on August 31, 2017

Reviewed on December 9, 2017

Accepted on December 22, 2017

Published online on December 7, 2018

Cite as

Kałużna M, Pawlaczyk K, Schwermer K, et al. Adropin and irisin: New biomarkers of cardiac status in patients with end-stage renal disease? A preliminary study. Adv Clin Exp Med. 2019;28(3):347-353. doi:10.17219/acem/81538

DOI

10.17219/acem/81538

Copyright

Copyright by Author(s)

This is an article distributed under the terms of the

Creative Commons Attribution Non-Commercial License

(http://creativecommons.org/licenses/by-nc-nd/4.0/)

\begin{abstract}
Background. The new polypeptide hormones adropin and irisin have a broad impact on human metabolism and energy homeostasis. They could be potential biomarkers of cardiac injury. In end-stage renal disease (ESRD), the clinical importance of adropin and irisin is yet to be investigated.
\end{abstract}

Objectives. The aim of this study was to determine the relationship between these peptides and cardiac status in ESRD patients.

Material and methods. Seventy-nine ESRD patients on hemodialysis (HD), peritoneal dialysis (PD) or after renal transplantation (TX), and 40 healthy, age- and sex-matched controls (CON) were included in this study. Serum concentrations of adropin and irisin were measured with enzyme-linked immunosorbent assay (ELLSA). Cardiac status was estimated by transthoracic echocardiography and the plasma concentration of $\mathrm{N}$-terminal pro-brain natriuretic peptide (NT-proBNP) and cardiac troponin T (CTnT).

Results. The levels of irisin were significantly lower in HD patients as compared to CON. During HD sessions, the concentrations of adropin did not change significantly, whereas the concentrations of irisin increased with borderline significance. Positive correlations were evident between adropin concentration and cTnT as well as NT-proBNP. Adropin was also correlated with left ventricular systolic internal diameter (LVIDs) $(r=0.375$, $p=0.045)$ and relative wall thickness (RWT) $(r=-0.382, p=0.034)$. Irisin was correlated with right ventricular diameter (RVd) $(r=-0.363, p=0.045)$. No correlations were found between irisin and adropin, and blood pressure (BP) measurements.

Conclusions. Adropin could be a new candidate marker of cardiac dysfunction in HD patients. The cause of low levels of irisin found in HD patients is still unclear. These 2 myokines should be further investigated as potential prognostic markers of cardiac status in HD patients.

Key words: hemodialysis, end-stage renal disease, irisin, adropin, cardiac status 


\section{Introduction}

End-stage renal disease (ESRD) leads to the development of a variety of hormonal and metabolic disorders. ${ }^{1}$ Myokines and adipokines, as regulators of metabolism, have recently gained increasing interest. Cardiovascular events remain the most common cause of death in patients treated with chronic hemodialysis (HD) or peritoneal dialysis (PD). ${ }^{2}$ The pathophysiology behind the increased relevance of cardiovascular disease (CVD) in dialyzed patients is still unclear. To prevent cardiac events and mortality, identification of high-risk cardiovascular patients is necessary. Therefore, determination of new and efficient markers of cardiovascular function in HD patients will be valuable.

Irisin and adropin, newly discovered peptide hormones, have broad implications in energy homeostasis and metabolism in humans. ${ }^{3-8}$ Adipokines and myokines can potentially provide a link between CVD and other features of metabolic syndrome (MS). Adropin is a polypeptide hormone that participates in the regulation of metabolism and energy homeostasis in humans. ${ }^{9}$ Recent data indicates that adropin contributes to the development of coronary atherosclerosis and heart failure (HF). ${ }^{5,10,11}$ This is supported by the fact that adropin appears to be a potential biomarker for endothelial dysfunction and CVD. ${ }^{11-19}$ Low plasma adropin levels have been reported in obesity, diabetes, insulin resistance, atherogenesis, cardiac syndrome X (CSX), and aging. ${ }^{6,10,13,18,19}$ The relationship between adropin level and kidney function has not been satisfactory established.

Irisin is a 112 amino acid protein of $12 \mathrm{kDa}$ molecular weight, and a product of fibronectin type III domain 5 (FNDC5) cleavage. ${ }^{20}$ Irisin converts white adipose tissue into brown adipose tissue, and is believed to improve glucose metabolism and repeal visceral obesity. ${ }^{20-22}$ Irisin has been proven to play a role in cardiomyoblast metabolism and blood pressure (BP) control. ${ }^{23,24}$ It has been proposed to be a possible marker of HF and myocardial infarction (MI). ${ }^{25-27}$ In contrast to other adipokines, irisin is not eliminated by renal excretion. ${ }^{28}$ Its concentration positively correlates with estimated glomerular filtration rate (eGFR), making it a potential predictor of chronic kidney disease development and progression. ${ }^{28,29}$ Interestingly, both irisin and adropin are produced locally in the heart. ${ }^{30,31}$

The aim of this study was to compare serum adropin and irisin levels in ESRD patients treated with HD, PD and kidney transplantation (Tx), and to determine the relationship between these peptides and cardiac status in HD patients. We hypothesized that adropin and irisin levels could be disturbed in ESRD patients and associated with cardiovascular complications and comorbidities, especially with altered cardiac function. We also expected that the level of these peptides could be altered by HD.

\section{Material and methods}

\section{Subjects}

Seventy-nine ESRD patients: 41 treated with HD (12 women, 29 men, median age 55 years, range $26-84$ years), 18 patients treated with continuous ambulatory PD (14 women, 4 men, median age 57 years, range $25-78$ years) and 20 ESRD patients after Tx (6 women, 14 men, median age 40.5 years, range $21-75$ years, $6-256$ months after Tx) at Heliodor Święcicki Clinical Hospital (Poznań, Poland) were recruited in January and February 2015 into this study. The exclusion criteria consisted of the following: diabetes mellitus or glucose intolerance, acute or chronic hepatic failure, hematologic disorders, active autoimmune disease or neoplasm, history of acute infection within the previous 7 days, and MI during the last 6 months. Forty healthy, age- and sex-matched people were also enrolled into the study as a control group (CON). The Bioethics Committee at Poznan University of Medical Sciences approved the study protocol. Patients gave their written informed consent to participate in the study.

\section{Blood chemistry measurements}

For HD patients, blood samples were collected before (HD1 group) and after the midweek HD (HD2 group) session, which means that ultimately, there were 5 groups of patients: HD1 group, HD2 group, PD group, Tx group, and CON. In all patients and controls, the sera were obtained following fasting (excluding HD patients after dialysis session) and stored at $-80^{\circ} \mathrm{C}$ until analysis. Serum levels of adropin were measured using enzyme-linked immunosorbent assay (ELISA) in duplicate and the results were averaged. The Human Adropin (ENHO) Elisa Kit (Cusabio Technology LLC, College Park, USA) was used. The sensitivity of this assay was below $0.39 \mathrm{pg} / \mathrm{mL}$. The same analytic method was used for serum irisin measurements with the AdipoGen Elisa Kit (AdipoGen Life Sciences, Liestal, Switzerland). The lowest detected concentration for this assay was $1 \mathrm{ng} / \mathrm{mL}$. In addition, serum creatinine, ferritin, N-terminal pro-brain natriuretic peptide (NT-proBNP), and cardiac troponin $\mathrm{T}$ (cTnT) were measured in HD patients prior to HD. These measurements were performed in the accredited and quality-controlled Central Laboratory of Heliodor Święcicki University Hospital, using NT-proBNP (pg/mL, Elecsys ${ }^{\circledR}$ assay, Roche Diagnostics, Basel, Switzerland) and cardiac troponin $\mathrm{T}$ levels (cTnT, Elecsys ${ }^{\circledast}$ Troponin $\mathrm{T}$ fourth generation assay, Roche Diagnostics, Basel, Switzerland).

\section{Examination of cardiovascular status}

Blood pressure measurements were performed in all HD patients prior to, during and after HD sessions. Cardiovascular function was assessed by 2 -dimensional (2-D), M-mode and Doppler echocardiography approx. $30 \mathrm{~min}$ 
after a routine HD session in 31 patients. ${ }^{32}$ The echocardiographic analyses were performed with a commercially available ultrasound system (M5S probe, Vivid E9; GE Healthcare, Milwaukee, USA). The M-mode and 2-D tracings were obtained with the subjects in the left lateral recumbent position, in accordance with the standards of the American Society of Echocardiography (ASE) and European Association of Cardiovascular Imaging (EACVI). ${ }^{33}$ Parasternal longand short-axis views, as well as apical 4- and 2-chamber views, were used for evaluation of the functions of the left ventricle (LV) and heart valves. One investigator performed all the echocardiographic examinations. Few patients did not agree to the echocardiographic examination. Endocardial border detection was enhanced by the use of second harmonic imaging. The left ventricular end-diastolic (LVEDd) and end-systolic (LVEDs) cavity dimensions, interventricular septal wall thickness (IVSd), left ventricular posterior wall thickness (LVPWTd), and left atrial dimension (LAD) were measured. Chamber systolic function was assessed as left ventricular fractional shortening (LVFS) at the endocardium. Left ventricular fractional shortening was calculated as:

$$
\text { LVFS }=[(\text { LVEDd }- \text { LVEDs }) / \text { LVEDd }] \times 100
$$

Left ventricular ejection fraction (LVEF) was calculated according to the Teichholz method. ${ }^{33}$ Left ventricular mass (LVM) was calculated using the American Society of Echocardiography formula modified by Devereux et al. ${ }^{34}$ :

$$
\begin{gathered}
\mathrm{LVM}=0.8 \times\left[1.04 \times\left(\mathrm{LVEDd}+\mathrm{IVSd}+\mathrm{LVPWTd}^{3}-\right.\right. \\
\left.-\mathrm{LVEDd}^{3}\right]+0.6 \mathrm{~g}
\end{gathered}
$$

The LV-mass index (LVMI) was determined by dividing the LVM by the body surface area of the patient. The upper normal limit is $115 \mathrm{~g} / \mathrm{m}^{2}$ for men and $95 \mathrm{~g} / \mathrm{m}^{2}$ for women. ${ }^{33}$

Doppler measurements of LV diastolic filling were performed by placing the pulsed Doppler sample volume at the mitral leaflet tips to obtain maximal velocities of early diastolic transmitral flow (E-wave peak velocity) and late transmitral flow (A-wave peak velocity). The E-wave deceleration time was measured, and the ratio between E- and A-wave peak velocities was subsequently derived. Mitral annular velocities at the septal corner and lateral corner of the mitral annulus were also recorded to determine peak early diastolic annular velocity. Left ventricle diastolic function was estimated according to EACVI recommendations on the basis of LV mitral inflow, peak early diastolic mitral annular velocity and the presence of left atrium (LA) enlargement. ${ }^{35}$

\section{Statistical analysis}

Statistical calculations were performed using STATISTICA v. 12 (StatSoft, Inc., Tulsa, USA). The Shapiro-Wilk test was used to determine whether the data followed
Table 1. Baseline characteristics of HD population - HD and laboratory parameters

\begin{tabular}{|l|c|}
\multicolumn{1}{|c|}{ Parameter } & Median (interquartile range) \\
\hline V urea $[\mathrm{L}]$ & $33.8(18.5-51.8)$ \\
\hline Kt/V & $1.44(0.65-1.84)$ \\
\hline Time of HD session $[\mathrm{min}]$ & $240(225-240)$ \\
\hline Ferritin $[\mu \mathrm{g} / \mathrm{L}]^{*}$ & $1545(155-3305)$ \\
\hline $\mathrm{CTnT}[\mathrm{ng} / \mathrm{L}]^{*}$ & $50(13-325)$ \\
\hline NT-proBNP $\left[\mathrm{pg} / \mathrm{mL}^{*}\right.$ & $4077(2330-35,000)$ \\
\hline
\end{tabular}

HD - hemodialysis; V urea - distribution volume of urea; Kt/V - number used to quantify hemodialysis treatment adequacy; $C T n T$ - cardiac troponin T; NT-proBNP - N-terminal pro-brain natriuretic peptide; * parameters measured prior to HD session.

a normal distribution. Results were expressed as median (interquartile range) and minimum-maximum range. The level of significance ( $\mathrm{p}$-value) was set below 0.05 for all analyses. In order to determine the differences between groups, the Kruskall-Wallis test was used. A possible association among measured parameters was quantified using Spearman's rank correlation coefficient $r$.

\section{Results}

\section{Clinical characteristic of hemodialysis patients}

The baseline characteristics of the HD population are summarized in Table 1. The HD vintage of patients varies from 92 to 7183 days. Hemodialysis patients had high levels of NTproBNP and cTnT. Of the 31 patients from the HD group in whom the echocardiography study was performed, the majority ( 9 women and 12 men) presented LV concentric hypertrophy. Two male patients had eccentric LV hypertrophy. In the remaining 8 patients, LV was normal. Normal LV systolic function, assessed by calculation of LVFS and LVEF, was found in 27 patients (7 women and 20 men). Abnormal LV relaxation was diagnosed in 3 patients ( 2 women and 1 man). The LA was enlarged in 27 patients, whereas 3 women and 1 man had normal LA diameter. The right ventricular dimension was normal in all examined patients (Table 2).

\section{Adropin and irisin before and after hemo- dialysis session, and in peritoneal dialysis, control and kidney transplantation group}

The levels of adropin did not change significantly before (HD1 group) and after (HD2 group) HD sessions (3.95 vs $4.26 \mathrm{ng} / \mathrm{mL}$, not significant (NS)) (Table 3). A negative correlation was observed between adropin concentrations and HD session duration $(\mathrm{r}=-0.421, \mathrm{p}=0.0085)$ (Table 4). There were no significant differences in the levels of adropin in all the groups - CON, PD, Tx, HD1, and HD2 (4.02 vs 4.74 vs 4.73 vs 3.95 vs $4.26 \mathrm{ng} / \mathrm{mL}$, NS) (Table 3 ). 
Table 2. Echocardiographic parameters in HD patients*

\begin{tabular}{|l|c|}
\hline \multicolumn{1}{|c|}{ Parameter } & Median (interquartile range) \\
\hline RVd $[\mathrm{mm}]$ & $14(22-36)$ \\
\hline IVSd $[\mathrm{mm}]$ & $13(10-27)$ \\
\hline PWd $[\mathrm{mm}]$ & $47(34-62)$ \\
\hline LAD $[\mathrm{mm}]$ & $30(20-44)$ \\
\hline LVIDs $[\mathrm{mm}]$ & $47(34-62)$ \\
\hline LVIDd $[\mathrm{mm}]$ & $36(17.02-50.00)$ \\
\hline LVFS $[\%]$ & $65.4(35.6-81.8)$ \\
\hline LVEF $[\%]$ & $03(0.39-1.04)$ \\
\hline RWT $[\mathrm{mm}]$ & $73(33-142)$ \\
\hline MVE $[\mathrm{cm} / \mathrm{s}]$ & $80.50(42-186)$ \\
\hline MV A $[\mathrm{cm} / \mathrm{s}]$ & $0.88(0.52-1.82)$ \\
\hline MVE/MVA [ms] & $200(130-485)$ \\
\hline MV E DT [ms] & $8.8(5-24)$ \\
\hline Mit E/E' $[\mathrm{ms}]$ & $272(133-744)$ \\
\hline LV mass [g] & $147(83-327)$ \\
\hline LV mass I [g/m²] &
\end{tabular}

HD - hemodialysis; RVd - right ventricular diameter; IVSd - interventricular septal end diastole dimension; PWd - posterior wall thickness at end-diastole; LAD - left atrium dimension; LVIDd - left ventricular internal diameter in diastole; LVIDs - left ventricular internal diameter in systole; RWT - relative wall thickness; LVFS - left ventricular fractional shortening; LVEF - left ventricular ejection fraction; MV E - mitral valve early (E) filling velocity; MV A - mitral valve atrial (A) filling velocity; MV E DT - deceleration time of mitral valve early (E) filling velocity; Mit $E / E^{\prime}$ - the ratio between early mitral inflow velocity and mitral annular early diastolic velocity; LV mass - left ventricular mass; LV mass I - left ventricular mass index; ${ }^{*}$ echocardiographic parameters were measured after HD session to avoid the influence of overhydration.

The median levels of irisin were significantly lower in $\mathrm{HD} 1$ patients compared to $\mathrm{CON}(4.32 \mathrm{vs} 7.85 \mu \mathrm{g} / \mathrm{mL}$; $\mathrm{p}<0.000001)$, PD patients $(4.32 \mathrm{vs} 9.73 \mu \mathrm{g} / \mathrm{mL}, \mathrm{p}=0.0015)$ and Tx patients $(4.32 \mathrm{vs} 6.96 \mu \mathrm{g} / \mathrm{mL}, \mathrm{p}<0.000001)$. The levels of irisin during HD sessions (HD1 vs HD2) increased with a borderline statistical significance (4.32 vs $5.01 \mu \mathrm{g} / \mathrm{mL}$; $\mathrm{p}=0.081$ ). There were no differences between levels of irisin in the Tx, PD and CON groups (Table 3). The detailed statistical analysis of adropin and irisin levels in all studied groups is presented in Table 3.

There was no correlation between irisin or adropin concentrations and HD vintage ( $\mathrm{p}<0.05)$, nor between adropin or irisin levels and time after $\mathrm{Tx}(\mathrm{p}<0.05)$.

\section{Cardiac status indicators}

Positive correlations were found between adropin preHD concentration and $\mathrm{cTnT}(\mathrm{r}=0.325, \mathrm{p}=0.046)$ as well as NT-proBNP ( $\mathrm{r}=0.355, \mathrm{p}=0.034)$ (Table 4). There was no correlation between adropin and BP measured before, during or after HD sessions. Irisin levels were not correlated with cTnT, NT-proBNP or BP.

Post-HD adropin levels were correlated to left ventricular internal systolic diameter (LVIDs) $(\mathrm{r}=0.375, \mathrm{p}=0.045)$ and relative wall thickness (RWT) $(\mathrm{r}=-0.382, \mathrm{p}=0.034)$. Post-HD irisin levels were correlated with RVd $(r=-0.363$, $\mathrm{p}=0.045)$. Absolute differences in adropin concentration before and after the midweek HD session were closely related to RVd ( $\mathrm{r}=-0.336, \mathrm{p}=0.064)$ (Table 4).

\section{Ferritin}

There was a negative correlation between pre-HD levels of irisin and ferritin $(\mathrm{r}=-0.335, \mathrm{p}=0.046)$ (Table 5). There was no correlation between adropin and ferritin concentration in HD patients.

\section{Discussion}

\section{Renal failure}

Adropin and irisin are hormones produced locally in many tissues. The renal metabolism of both myokines is not well understood. So far adropin has been isolated

Table 3. Adropin and irisin levels in studied groups. Results are expressed as median (min-max range)

\begin{tabular}{|c|c|c|c|c|}
\hline $\begin{array}{l}\text { Groups chosen } \\
\text { for comparison }\end{array}$ & $\begin{array}{l}\text { Adropin }[\mathrm{ng} / \mathrm{mL}] \text { median concentration } \\
\text { (interquartile range) for both groups }\end{array}$ & $\mathrm{p}$-value & Irisin [ug/mL] & p-value \\
\hline HD1 vs CON & $3.95(1.49-6.41)$ vs $4.02(1.23-7.84)$ & NS & $4.32(2.51-11.95)$ vs $7.85(5.34-16.80)$ & $p<0.0001$ \\
\hline HD1 vs HD2 & $3.95(1.49-6.41)$ vs $4.26(1.67-7.23)$ & NS & $4.32(2.51-11.95)$ vs $5.01(3.20-14.11)$ & NS \\
\hline HD1 vs PD & $3.95(1.49-6.41)$ vs $4.73(2.64-8.50)$ & NS & $4.32(2.51-11.95)$ vs $6.96(4.50-13.55)$ & $p=0.0015$ \\
\hline HD1 vs Tx & $3.95(1.49-6.41)$ vs $4.74(2.76-9.01)$ & NS & $4.32(2.51-11.95)$ vs $9.73(6.27-20.00)$ & $p<0.0001$ \\
\hline HD2 vs CON & $4.26(1.67-7.23)$ vs $4.02(1.23-7.84)$ & NS & $5.01(3.20-14.11)$ vs $7.85(5.34-16.80)$ & $p<0.0001$ \\
\hline HD2 vs PD & $4.26(1.67-7.23)$ vs $4.73(2.64-8.50)$ & NS & $5.01(3.20-14.11)$ vs $6.96(4.50-13.55)$ & NS \\
\hline HD2 vs Tx & $4.26(1.67-7.23)$ vs $4.74(2.76-9.01)$ & NS & $5.01(3.20-14.11)$ vs $9.73(6.27-20.00)$ & $p<0.0001$ \\
\hline PD vs CON & $4.73(2.64-8.50)$ vs $4.02(1.23-7.84)$ & NS & $6.96(4.50-13.55)$ vs $7.85(5.34-16.80)$ & NS \\
\hline Tx vs CON & $4.74(2.76-9.01)$ vs $4.02(1.23-7.84)$ & NS & $9.73(6.27-20.00)$ vs $7.85(5.34-16.80)$ & NS \\
\hline PD vs Tx & $4.73(2.64-8.50)$ vs $4.74(2.76-9.01)$ & NS & $6.96(4.50-13.55)$ vs $9.73(6.27-20.00)$ & NS \\
\hline
\end{tabular}

HD - hemodialysis; CON - median value in healthy controls; HD1 - median value prior to HD session; HD2 - median value after HD session;

PD - median value in peritoneal dialysis patients; Tx - median value in patients after renal transplantation; NS - not significant. 
Table 4. Correlation coefficient $r$ between serum adropin concentration and $\mathrm{HD}$, laboratory and ECHO parameters

\begin{tabular}{|l|c|c|}
\hline \multicolumn{1}{|c|}{ Variables } & Adropin prior to HD & Adropin after HD \\
\hline V urea & $r=-0.707, p<0.0001$ & $r=-0.587, p=0.0002$ \\
\hline Kt/v & $r=0.308$, NS & $r=0.039$, NS \\
\hline HD session duration & $r=-0.421, p=0.0085$ & $r=-0.403, p=0.012$ \\
\hline Ferritin & - & $r=0.117$, NS \\
\hline CTnT & - & $r=0.325, p=0.0460$ \\
\hline NT-proBNP & - & $r=0.496, p=0.0020$ \\
\hline RVd & - & $r=-0.201, N S$ \\
\hline LVIDs & - & $r=0.375, p<0.0001$ \\
\hline RWT & - & $r=0.382, p=0.0340$ \\
\hline
\end{tabular}

HD - hemodialysis; V urea - distribution volume of urea; Kt/V - number used to quantify hemodialysis treatment adequacy; CTn-T - cardiac troponin-T; NT-proBNP - N-terminal pro-brain natriuretic peptide; RVd - right ventricular diameter; LVIDs - left ventricular internal diameter in systole; RWT - relative wall thickness; $r$ - correlation coefficient; NS - not significant.

Table 5. Correlation coefficient $r$ between serum irisin concentration and $\mathrm{HD}$ parameters, laboratory and ECHO parameters

\begin{tabular}{|c|c|c|}
\hline Variables & Irisin prior to HD & Irisin after HD \\
\hline Vurea & $r=0.026, \mathrm{NS}$ & $r=-0.168$, NS \\
\hline $\mathrm{Kt} / \mathrm{V}$ & $r=-0.215, \mathrm{NS}$ & $r=-0.061, \mathrm{NS}$ \\
\hline HD session duration & $r=0.007$, NS & $r=0.116, N S$ \\
\hline Ferritin & $r=-0.335, p=0.0460$ & $r=-0.252, \mathrm{NS}$ \\
\hline cTnT & - & $r=0.027, \mathrm{NS}$ \\
\hline NT-proBNP & - & $r=0.102, \mathrm{NS}$ \\
\hline RVd & - & $r=-0.363, p=0.0450$ \\
\hline LVIDs & - & $r=-0.025, N S$ \\
\hline RWT & - & $r=-0.116$, NS \\
\hline
\end{tabular}

HD - hemodialysis; V urea - distribution volume of urea; Kt/V - number used to quantify hemodialysis treatment adequacy; $C T n-T$ - cardiac troponin-T; NT-proBNP - N-terminal pro-brain natriuretic peptide; RVd - right ventricular diameter; LVIDs - left ventricular internal diameter in systole; RWT - relative wall thickness; $r$ - correlation coefficient; NS - not significant.

in the liver, muscle, intestines, kidney, heart, pancreas, brain, umbilical vein, and salivary glands. ${ }^{6,9,19,31,36,37}$ In rats with isoproterenol (ISO)-induced MI, the expression of adropin dramatically increased in the kidney tissues. ${ }^{12}$ Therefore, we hypothesized that the adropin levels might be altered in HD patients, as renal insufficiency is usually accompanied by CVD. However, we found no statistically significant difference in adropin levels when comparing HD patients and CON. Also, no statistically significant difference was observed between the adropin level prior to and after the HD session. The results of our study revealed that adropin is dialyzable during HD. Changes of adropin level should be interpreted in accordance to eGFR. However, the low sample size of HD and PD patients could have interfered with the results of the study.

We found a borderline statistically significant rise in irisin level during HD sessions, which was not related to UF and/or HD adequacy measured by Kt/V. This is not consistent with the recent results of Ebert et al., who found a significant $23 \%$ decrease in irisin level after HD. ${ }^{28}$ The increase in concentration in our study could be attributed to decreased intravascular volume; however, this finding questions the dialyzable nature of irisin. Hemodialysis itself does not seem to be the reason for low irisin concentrations in HD patients. A study by Wen et al. showed that uremic toxins have a negative influence on FNDC5 expression, which is the precursor of irisin. ${ }^{29}$ Studies using larger samples are required to validate the dialyzable nature of irisin.

Other factors such as decreased physical activity, malnutrition and cachexia in HD patients could be the possible reasons of the low irisin level in the group of HD patients. However, just as our results, finding on these topics are inconclusive. Perhaps the negative correlation between irisin and ferritin, a marker of iron stores in dialysis patients and a positive acute-phase reactant in our study may show a connection between chronic inflammation and low irisin levels in HD patients. The pathophysiological significance of low irisin levels in HD patients needs to be further investigated.

\section{Cardiac function}

Cardiac dysfunction is accompanied by a number of changes in the neuroendocrine system. ${ }^{38}$ Adropin immunoreactivity was found in the endocardium, myocardium and epicardium. ${ }^{31}$ In our study, there was a significant positive correlation between adropin level, cTnT and NTproBNP. These findings demonstrate a probable and complex relation between adropin concentration and the cardiac status of HD patients. Many studies have displayed the role of cTnT and NT-proBNP as significant predictors of cardiovascular and all-cause mortality in ESRD patients. ${ }^{39-41}$ However, in ESRD patients, interpretation of cTnT and NT-proBNP can be confusing as an elevation of these biochemical parameters can be the effect of renal insufficiency and overhydration.

The available data on the relationship between adropin and altered cardiac function is inconsistent. Lian et al. found that patients with HF are characterized by higher adropin levels than healthy controls and implied a potential role of high adropin levels in the development of the HF. ${ }^{11}$ The elevation in adropin was proportional to the severity of the heart disease according to New York Heart Association (NYHA) classification. ${ }^{11}$ A negative relationship between adropin and LVEF $(r=-0.710, p<0.001)$ was also observed in their study. ${ }^{11}$ In our study, we did not observe any correlations between adropin and LVEF after HD. This could be attributed to our sample, as the majority of the HD patients had normal LV systolic function and only 4 patients had diminished LVEF. We found a positive correlation between adropin concentration and LVIDs and a negative correlation between irisin and RVd. The correlation 
between adropin level and RWT observed in our study may suggest that adropin concentration is related to concentric LV hypertrophy; however, we did not observe any correlations between adropin and the LVMI. To establish this fact, further studies with a larger and more diversified group of patients regarding LV function is needed.

Other researchers have noted that increased adropin concentration could be a potential biomarker of cardiac status. Aydin et al. in their animal study, proposed using elevated adropin levels in conjunction with elevated troponin as biomarkers of MI. ${ }^{12}$ The synthesis of adropin in rats was increased within $24 \mathrm{~h}$ after MI (induced by isoproterenol) in most tissues, including cardiac muscle cells. ${ }^{12}$ However, opposing results were observed in humans. Serum adropin level in patients with MI was significantly lower in comparison to subjects with stable angina pectoris or controls in a study by Yu et al. ${ }^{15}$ Furthermore, lower adropin level was found to be an independent predictor for MI in coronary artery disease (CAD) patients. ${ }^{15}$ Interestingly, no correlation between serum adropin levels and cTnT or NT-proBNP was observed, ${ }^{15}$ contrasting with the findings of our study.

A decrease in adropin concentration is considered as a new marker of coronary atherosclerosis. ${ }^{10,18,42,43}$ The data suggests low serum adropin level as a predictor of stable CAD, and an association with the severity of coronary atherosclerosis. ${ }^{17,44}$ Low concentrations of adropin were described to be related to coronary atherosclerosis in both diabetic and non-diabetic groups of patients. ${ }^{42}$ Lower serum adropin level was also found to be an independent risk factor for CSX. ${ }^{13}$

Conflicting data on levels of adropin in cardiac dysfunction most likely suggests a complex role of adropin in different types of CVD and varying groups of patients. Factors such as concomitant diseases or additional hormonal and metabolic factors that could have overlapped with the study cannot be excluded. The diagnostic and prognostic value of adropin in the field of CVD and events in ESRD patients is still unclear. We cannot extrapolate the results from animal studies on adropin to humans. The relationship between adropin level and cardiac dysfunction in ESRD patients requires further investigation in order to consider it as a cardiac biomarker and improve cardiovascular risk assessment of ESRD patients in the future.

Low irisin levels were described in patients with HF. ${ }^{25,26}$ We reported decreased irisin levels in HD patients. Lack of irisin upregulation in insulin resistance and other components of metabolic syndrome might hypothetically suggest the development of underlying cardiac insufficiency in ESRD patients treated with HD. However, we did not find a relation between irisin and cardiac markers in the subjects examined. This could be attributed to the small sample or the fact that the majority of our subjects had normal LV systolic function.

The main limitation of this study was the small number of patients included. The small sample size in our study may have prevented our results from reaching a more significant level. Further prospective longitudinal studies with a larger number of patients are needed to highlight the role of adropin in the metabolic homeostasis of ESRD patients.

\section{Conclusions}

Hormonal imbalances can be both the reason and the result of advancing ESRD and its complications. This study is the first to indicate an association between the levels of 2 myokines, adropin and irisin simultaneously, and the cardiac status of HD patients. ${ }^{31}$ The significant positive correlation between adropin, cTnT and NT-proBNP demonstrates a probable and complex relation between adropin level and the cardiac status of HD patients. Our findings suggest that adropin may be involved in the pathogenesis of cardiac dysfunction in HD patients, but it certainly needs further detailed analysis and studies on the pathogenic mechanism. Of the 3 groups studied (HD, PD and Tx), HD patients had the lowest concentration of irisin. Nevertheless, adropin and irisin have to be interpreted carefully with regard to the patient's individual residual renal function and concomitant diseases. The new myokines, especially adropin, should be under further investigation as potential prognostic markers of cardiac status and clinical status in HD patients.

\section{References}

1. Herzog CA, Asinger RW, Berger AK, et al. Cardiovascular disease in chronic kidney disease. A clinical update from Kidney Disease: Improving Global Outcomes (KDIGO). Kidney Int. 2011;80(6):572-586.

2. Ruchala M, Zybek A, Szczepanek-Parulska E. Serum irisin levels and thyroid function: Newly discovered association. Peptides. 2014;60:51-55.

3. Zybek-Kocik A, Sawicka-Gutaj N, Wrotkowska E, Sowiński J, Ruchala M. Time-dependent irisin concentration changes in patients affected by overt hypothyroidism. Endokrynol Pol. 2016;67(5):476-480.

4. Aydin S. Three new players in energy regulation: Preptin, adropin and irisin. Peptides. 2014;56:94-110.

5. Butler AA, Tam CS, Stanhope KL, et al. Low circulating adropin concentrations with obesity and aging correlate with risk factors for metabolic disease and increase after gastric bypass surgery in humans. J Clin Endocrinol Metab. 2012;97(10):3783-3791.

6. Chen M, Ouyang F, Zhou S. Adropin as a novel energy factor likely has the ability to regulate blood pressure. Med Hypotheses. 2015;85(2):234

7. Colaianni G, Cuscito $C$, Mongelli T, et al. The myokine irisin increases cortical bone mass. Proc Natl Acad Sci US A. 2015;112(39):12157-12162.

8. Kumar KG, Trevaskis JL, Lam DD, et al. Identification of adropin as a secreted factor linking dietary macronutrient intake with energy homeostasis and lipid metabolism. Cell Metab. 2008;8(6):468-481.

9. Wu L, Fang J, Chen L, et al. Low serum adropin is associated with coronary atherosclerosis in type 2 diabetic and non-diabetic patients. Clin Chem Lab Med. 2014;52(5):751-758.

10. Lian W, Gu X, Qin Y, Zheng X. Elevated plasma levels of adropin in heart failure patients. Intern Med. 2011;50(15):1523-1527.

11. Aydin S, Kuloglu T, Aydin S, et al. Elevated adropin: A candidate diagnostic marker for myocardial infarction in conjunction with troponin-I. Peptides. 2014;58:91-97.

12. Celik A, Balin M, Kobat MA, et al. Deficiency of a new protein associated with cardiac syndrome $\mathrm{X}$ called adropin. Cardiovasc Ther. 2013;31(3):174-178.

13. Goetze JP, Albrethsen J. Adropin: A new regulatory peptide in cardiovascular endocrinology. Regul Pept. 2014;190-191:41-42. 
14. Yu HY, Zhao P, Wu MC, Liu J, Yin W. Serum adropin levels are decreased in patients with acute myocardial infarction. Regul Pept. 2014;190-191:46-49.

15. Zhang $C$, Zhao L, Xu W, et al. Correlation of serum adropin level with coronary artery disease [in Chinese]. Zhonghua Yi Xue Za Zhi. 2014;94(16):1255-1257.

16. Zhao LP, Xu WT, Wang L, et al. Serum adropin level in patients with stable coronary artery disease. Heart Lung Circ. 2015;24(10):975-979.

17. Topuz M, Celik A, Aslantas T, Demir AK, Aydin S, Aydin S. Plasma adropin levels predict endothelial dysfunction like flow-mediated dilatation in patients with type 2 diabetes mellitus. $J$ Investig Med. 2013;61(8):1161-1164.

18. Lovren F, Pan Y, Quan A, et al. Adropin is a novel regulator of endothelial function. Circulation. 2010;122(11 Suppl):S185-192.

19. Bostrom $P, W u$ J, Jędrychowski MP, et al. A PGC1-alpha-dependent myokine that drives brown-fat-like development of white fat and thermogenesis. Nature. 2012;481(7382):463-468.

20. Lopez-Legarrea $P$, de la Iglesia $R$, Crujeiras $A B$, et al. Higher baseline irisin concentrations are associated with greater reductions in glycemia and insulinemia after weight loss in obese subjects. Nutr Diabetes. 2014;4(2):e110.

21. Vamvini MT, Aronis KN, Panagiotou G, et al. Irisin mRNA and circulating levels in relation to other myokines in healthy and morbidly obese humans. Eur J Endocrinol. 2013;169(6):829-834.

22. Xie C, Zhang Y, Tran TD, et al. Irisin controls growth, intracellular $\mathrm{Ca}^{2+}$ signals, and mitochondrial thermogenesis in cardiomyoblasts. PLoS One. 2015;10(8):e0136816.

23. Zhang W, Chang L, Zhang $C$, et al. Central and peripheral irisin differentially regulate blood pressure. Cardiovasc Drugs Ther. 2015;29(2):121-127.

24. Emanuele E, Minoretti P, Pareja-Galeano H, Sanchis-Gomar F, Garatachea N, Lucia A. Serum irisin levels, precocious myocardial infarction and healthy exceptional longevity. Am J Med. 2014;127(9):888-890.

25. Lecker $\mathrm{SH}$, Zavin A, Cao $\mathrm{P}$, et al. Expression of the irisin precursor FNDC5 in skeletal muscle correlates with aerobic exercise performance in patients with heart failure. Circ Heart Fail. 2012;5(6):812-818.

26. Kuloglu T, Aydin S, Eren MN, et al. Irisin: A potentially candidate marker for myocardial infarction. Peptides. 2014;55:85-91.

27. Ebert T, Focke D, Petroff D, et al. Serum levels of the myokine irisin in relation to metabolic and renal function. Eur J Endocrinol. 2014;170(4):501-506.

28. Wen MS, Wang CY, Lin SL, Hung KC. Decrease in irisin in patients with chronic kidney disease. PLoS One. 2013;8(5):e64025.

29. Aydin S, Kuloglu T, Aydin S, et al. Cardiac, skeletal muscle and serum irisin responses to with or without water exercise in young and old male rats: Cardiac muscle produces more irisin than skeletal muscle. Peptides. 2014;52:68-73.
30. Aydin S, Kuloglu T, Aydin S, et al. Expression of adropin in rat brain, cerebellum, kidneys, heart, liver, and pancreas in streptozotocininduced diabetes. Mol Cell Biochem. 2013;380(1-2):73-81.

31. Lang RM, Bierig M, Devereux RB, et al; Chamber Quantification Writing Group; American Society of Echocardiography's Guidelines and Standards Committee; European Association of Echocardiography. Recommendations for chamber quantification. Eur J Echocardiogr. 2006;7(2):79-108.

32. Lang RM, Badano LP, Mor-Avi V, et al. Recommendations for cardiac chamber quantification by echocardiography in adults: An update from the American Society of Echocardiography and the European Association of Cardiovascular Imaging. Eur Heart J Cardiovasc Imaging. 2015;16(3):233-270.

33. Devereux RB, Alonso DR, Lutas EM, et al. Echocardiographic assessment of left ventricular hypertrophy: Comparison to necropsy findings. Am J Cardiol. 1986;57(6):450-458.

34. Nagueh SF, Appleton CP, Gillebert TC, et al. Recommendations for the evaluation of left ventricular diastolic function by echocardiography. J Am Soc Echocardiogr. 2009;22(2):107-133.

35. Wang SP, Gao YL, Liu G, et al. Molecular cloning, characterization and expression of the energy homeostasis-associated gene in piglet. J Zhejiang Univ Sci B. 2015;16(6):524-532.

36. Ganesh Kumar K, Zhang J, Gao S, et al. Adropin deficiency is associated with increased adiposity and insulin resistance. Obesity (Silver Spring). 2012;20(7):1394-1402.

37. Martin MW. Treatment of congestive heart failure - a neuroendocrine disorder. J Small Anim Pract. 2003;44(4):154-160.

38. Oh HJ, Lee MJ, Lee HS, et al. NT-proBNP: Is it a more significant risk factor for mortality than troponin $\mathrm{T}$ in incident hemodialysis patients? Medicine (Baltimore). 2014;93(27):e241.

39. Schwermer K, Hoppe K, Radziszewska D, et al. N-terminal proBtype natriuretic peptide as a marker of hypervolemia and predictor of increased mortality in patients on hemodialysis. Pol Arch Med Wewn. 2015;125(7-8):560-569.

40. Hoppe K, Schwermer K, Kłysz P, et al. Cardiac troponin T and hydration status as prognostic markers in hemodialysis patients. Blood Purif. 2015;40(2):139-145.

41. St-Onge M-P, Shechter A, Shlisky J, et al. Fasting plasma adropin concentrations correlate with fat consumption in human females. Obesity (Silver Spring). 2014;22(4):1056-1063.

42. Celik E, Yilmaz E, Celik O, et al. Maternal and fetal adropin levels in gestational diabetes mellitus. J Perinat Med. 2013;41(4):375-380.

43. Bornfeldt $\mathrm{KE}$, Tabas I. Insulin resistance, hyperglycemia and atherosclerosis. Cell Metab. 2011;14(5):575-585. 